
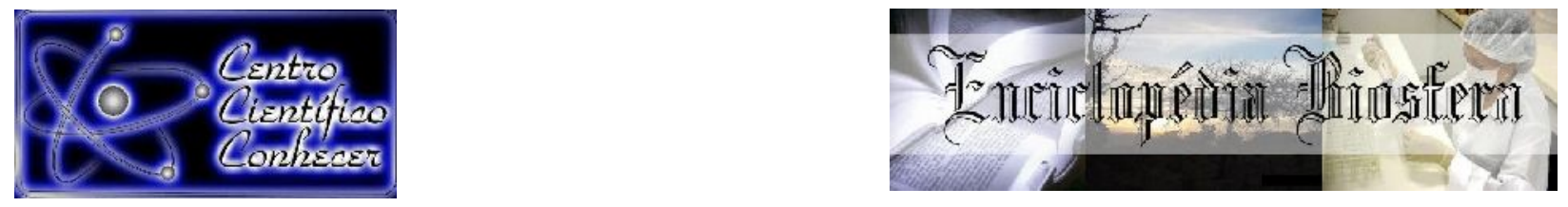

\title{
ANÁLISE DAS INFORMAÇÕES CONTIDAS NOS RÓTULOS E DO CONSUMO DE DIFERENTES MARCAS DE ÁGUA MINERAL E REFRIGERANTES
}

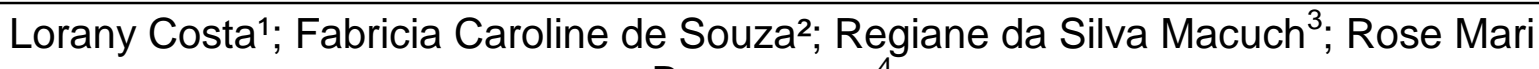
Bennemann ${ }^{4}$

'Discente do Curso de Nutrição, Centro Universitário de Maringá - UNICESUMAR, Maringá, Paraná, Brasil. Bolsista PROBIC/UniCesumar. (Ioranycosta@hotmail.com).

${ }^{2}$ Discente do Curso de Nutrição, Centro Universitário de Maringá - UNICESUMAR, Maringá, Paraná, Brasil.

${ }^{3}$ Professora Doutora, do Programa de Mestrado em Promoção da Saúde do Centro

Universitário de Maringá - UNICESUMAR e pesquisadora do Instituto Cesumar de Ciência, Tecnologia e Inovação - ICETI, Maringá, Paraná, Brasil.

${ }^{4}$ Orientadora, Professora Doutora, do Programa de Mestrado em Promoção da Saúde do Centro Universitário de Maringá - UNICESUMAR e pesquisadora do Instituto Cesumar de Ciência, Tecnologia e Inovação - ICETI, Maringá, Paraná, Brasil.

Recebido em: 22/09/2018 - Aprovado em: 23/11/2018 - Publicado em: 03/12/2018 DOI: 10.18677/EnciBio_2018B92

\section{RESUMO}

A água é fundamental para que o corpo humano possa realizar as funções vitais. A recomendação de ingestão adequada (Al) para indivíduos entre 19 a 70 anos é de 2,7L para mulheres e 3,7L para homens. A ingestão de água engarrafada vem crescendo gradualmente no Brasil. Em sua composição está presente 0 mineral sódio, que, embora seja fundamental para a realização das funções vitais do organismo, em excesso, pode levar ao desenvolvimento de doenças crônicas não transmissíveis (DCNTs). Em refrigerantes encontra-se presente o sódio e também açúcares. A recomendação máxima de ingestão de sódio é de $2 \mathrm{~g} / \mathrm{dia}$ e a recomendação de açúcar não deve ultrapassar $10 \%$ do valor energético total, cerca de $50 \mathrm{~g}$ para uma dieta de $2.000 \mathrm{kcal} / \mathrm{dia}$. Este estudo objetivou analisar as informações contidas nos rótulos e o consumo de diferentes marcas de água mineral e refrigerantes. As três marcas de água engarrafada que apresentaram maiores teores de sódio por litro, em ordem decrescente, foram: Badoit $\AA(180,0 \mathrm{mg})$, Sferrie $\AA$ $(75,8 \mathrm{mg})$ e São Lourenço® $(36,0 \mathrm{mg})$. As três marcas de refrigerante que apresentaram maiores teores de sódio a cada $200 \mathrm{~mL}$ de produto, por ordem decrescente foram: Fanta Laranja $\AA$ (77mg), Schweppes Citrus $\AA^{\circledR}(50 \mathrm{mg})$ e Sprite Ice Mint ${ }^{\circledR}(38 \mathrm{mg})$. As marcas de refrigerante que apresentaram teores de açúcares superiores a $40 \%$ das recomendações diárias de ingestão, ou seja, acima de $20 \mathrm{~g}$, em ordem decrescente, foram: Ouro Verde Limão $\AA$, com $24 \mathrm{~g}$; Ouro Verde Citrus $\AA$ e Pepsi®, com 22g cada; e Coca Cola $\AA$, Fanta Guaraná $\AA^{\circledR}$ e Sprite $\AA$, com 21 g cada.

PALAVRAS-CHAVE: Alimentação; DCNTs; Saúde. 


\title{
ANALYSIS OF INFORMATION CONTAINED IN THE LABELS AND CONSUMPTION OF DIFFERENT MINERAL WATER AND REFRIGERANT MARKS
}

\begin{abstract}
Water is fundamental so that the human body can perform vital functions. The recommended intake $(\mathrm{Al})$ for individuals aged $19-70$ is $2.7 \mathrm{~L}$ for women and $3.7 \mathrm{~L}$ for men. Intake of bottled water has been increasing gradually in Brazil. Mineral sodium is present in its composition, which, although is essential to the performance of vital functions of the body, in excess, may lead to the development of chronic noncommunicable diseases (NCDs). In soft drinks, sodium and sugars are present. The maximum recommendation for sodium intake is $2 \mathrm{~g} /$ day and the sugar recommendation should not exceed $10 \%$ of the total energy value, about $50 \mathrm{~g}$ for a $2,000 \mathrm{kcal} /$ day diet. This study aimed to analyze the information contained in the labels and the consumption of different brands of mineral water and soft drinks. The three brands of bottled water that presented the highest levels of sodium per liter in descending order were: Badoit ${ }^{\circledR}(180.0 \mathrm{mg})$, Sferrie ${ }^{\circledR}(75.8 \mathrm{mg})$ and São Lourenço® $(36.0 \mathrm{mg})$. The three brands of soft drinks that presented the highest sodium content per $200 \mathrm{ml}$ of product, in descending order were: Fanta Laranja ${ }^{\circledR}(77 \mathrm{mg})$, Schweppes Citrus ${ }^{\circledR}(50 \mathrm{mg})$ and Sprite Ice Mint ${ }^{\circledR}(38 \mathrm{mg})$. The brands of soft drinks that presented sugar content higher than $40 \%$ of daily intake recommendations, that is, above $20 \mathrm{~g}$, in decreasing order were: Ouro Verde Limãoß, with 24g; Gold Green Citrus $\AA$ and Pepsi $\AA$, with $22 \mathrm{~g}$ each; and Coca Cola $\AA$, Fanta Guaraná ${ }^{\circledR}$ and Sprite $\AA$, with $21 \mathrm{~g}$ each.
\end{abstract}

KEYWORDS: Food; NCDs; Health.

\section{INTRODUÇÃO}

A água pode ser definida como o mais importante e mais abundante composto inorgânico em todos os sistemas vivos e em adultos magros constitui cerca de 55 a $60 \%$ do corpo (TORTORA; DERRICKSON, 2016). A água é fundamental para que o corpo humano possa realizar as funções vitais. A recomendação da ingestão, segundo Couto et al., (2014), é de pelo menos dois litros por dia. Já as recomendações das Dietary Reference Intakes (DRIs), para adultos de 19 a 70 anos de idade, a ingestão adequada (Al) é de 2,7L para mulheres e 3,7L para homens (NAP, 2005).

A ingestão de água engarrafada vem crescendo gradualmente em diversos países. De 2012 a 2017 o consumo de água na China aumentou 11,8\%, na Indonésia 10,4\%, na Índia 9,7\%, nos Estados Unidos 7,1\% e na Tailândia 4,8\% (RODWAN Jr, 2017). No Brasil, em 2012 o consumo de água engarrafada era de aproximadamente 17 bilhões de litros (RODWAN Jr, 2017), em 2013 de 18 bilhões de litros, em 2014 de 19,5 bilhões de litros (BRASIL, 2016a). Em 2017 o consumo aproximado já era de 22 bilhões de litros, correspondendo, no período de 2012 a 2017, ao aumento de 4,7\% do consumo de água engarrafada (RODWAN Jr, 2017).

A água mineral natural, de acordo com a RDC 54/2000, é aquela que contém conteúdo constante de sais minerais, oligoelementos e outros constituintes e é obtida diretamente de fontes naturais ou artificialmente captadas, de origem subterrânea (ANVISA, 2000). Dentre as diversas substâncias contidas na água engarrafada, um dos minerais que mais encontra-se presente é o sódio. No corpo humano o sódio $\left(\mathrm{Na}^{+}\right)$, segundo Franco (2001), é fundamental para regulação osmótica e passagem do impulso nervoso, que são importantes funções celulares. 
Dados do Ministério da Saúde apontam que, a ingestão de $\mathrm{Na}^{+}$pela população brasileira, em 2016, era de 4,8g por pessoa por dia (12g de sal) (BRASIL, 2016b), sendo 2,4 vezes acima da recomendação estabelecida pela World and Health Organization (WHO, 2012) que era em 2012, de $2 \mathrm{~g}$ de $\mathrm{Na}^{+}$por dia, ou menos de $5 \mathrm{~g}$ de sal, para uma pessoa adulta.

A ingestão excessiva de $\mathrm{Na}^{+}$tem sido correlacionada com o desenvolvimento de doenças crônicas não-transmissíveis (DCNTs), como doenças cardiovasculares, sendo esta uma das principais causas de morte prematura no mundo (CLARO et al., 2015; AFSHIN et al., 2017). Segundo a WHO, em 2016, a prevalência de mortalidade mundial por DCNTs foi $71 \%$ do total de mortes, equivalente a 40,5 milhões de pessoas (WHO, 2018a). No Brasil, em 2011, as DCNTs corresponderam a 72,6\% das causas de morte, em 2015 foram 75,8\% (MALTA et al., 2015) e em 2016 foram 74\% das causas de morte (WHO, 2018b).

Para combater as DCNTs o Brasil, em 2010, criou o plano de ações estratégicas para o enfrentamento das DCNTs, que tem como meta a redução da ingestão de $\mathrm{Na}^{+}$pela população brasileira de 4,8g (12g de sal) em 2010 para $2 \mathrm{~g}$ (5g de sal) até 2022 (MALTA; SILVA Jr, 2013; BRASIL, 2018a).

Os benefícios da redução da ingestão de $\mathrm{Na}^{+}$, segundo o Ministério da Saúde levará a diminuição de $15 \%$ dos óbitos por acidente vascular cerebral (AVC) (100.050 óbitos em 2013), redução de 10\% de óbitos por infarto (85.939 mortes em 2013), 1,5 milhão de pessoas livres de medicação para hipertensão e quatro anos a mais na expectativa de vida de indivíduos hipertensos (BRASIL, 2016b). Na indústria a adição de $\mathrm{Na}^{+}$nos alimentos tem como finalidade aumentar a vida de prateleira do produto e pode ser encontrado tanto em alimentos quanto em bebidas e em refrigerantes.

Além da água, o refrigerante encontra-se amplamente presente nas mesas da população brasileira. Segundo a pesquisa da vigilância de fatores de risco e proteção para doenças crônicas por inquérito telefônico (VIGITEL), em 2017, a frequência de consumo de refrigerante foi de $14,6 \%$ em cinco ou mais dias da semana (BRASIL, 2018a). Além do $\mathrm{Na}^{+}$, encontra-se outros nutrientes nos refrigerantes, como os carboidratos $(\mathrm{CHO})$ simples, chamados também de açúcares.

Assim como o $\mathrm{Na}^{+}$, os $\mathrm{CHO}$ são essenciais para o funcionamento do organismo, pois fornecem energia para o funcionamento celular, especialmente para o cérebro, que é um órgão glicose-dependente (CUPPARI, 2014). Para Silva e Mura (2016), os carboidratos são compostos extremamente abundantes na natureza e representam cerca de $60 \%$ das necessidades energéticas da população dos países em desenvolvimento.

A recomendação de ingestão de $\mathrm{CHO}$ para uma pessoa adulta de acordo com a RDA é de no mínimo $130 \mathrm{~g} /$ dia para manter as funções cerebrais (NAP, 2005). A recomendação de ingestão de $\mathrm{CHO}$ deve estar entre 45 a $65 \%$ da recomendação energética total (CUPPARI, 2014) e a ingestão de açúcares simples não deve ser superior a $10 \%$ do valor energético total, cerca de $50 \mathrm{~g}$ ou $200 \mathrm{kcal}$ para uma dieta de $2.000 \mathrm{kcal} / \mathrm{dia}$ (BRASIL, 2016b). O excesso da ingestão de $\mathrm{CHO}$ pode gerar balanço calórico positivo, se a ingestão for superior a perda de calorias, acarretando ganho de peso, podendo levar à obesidade (CUPPARI, 2014).

De acordo com levantamento realizado, em diversos países, pela World Health Organization, em 2016, 39\% dos adultos estavam acima do peso e 13\% estavam obesos (WHO, 2018c). No Brasil a pesquisa realizada pela VIGITEL em 26 capitais brasileiras e no Distrito Federal, em 2017, verificou que 54,0\% da população encontrava-se com excesso de peso e 18,9\% com obesidade (BRASIL, 2018b). 
Nesse sentido, o objetivo do presente estudo foi analisar as informações contidas nos rótulos e o consumo de diferentes marcas de água mineral e refrigerantes.

\section{MATERIAL E MÉTODOS}

O estudo foi de natureza quantitativa, transversal, com coleta de dados primários e secundários. Foram visitados quatro supermercados de redes de supermercados, na cidade de Maringá - PR. Foram verificadas as marcas e anotados, em formulário próprio, os teores de sódio, de carboidrato, a marca, bem como o número do lote correspondente das garrafas de água e refrigerante. Foram pesquisadas 17 marcas de água engarrafada e 21 marcas de refrigerantes.

Para as entrevistas foram convidados a participar 65 indivíduos, de ambos os sexos, com idade entre 19 a 70 anos, que estavam adquirindo água engarrafada ou refrigerantes nos supermercados. Foram excluídos do estudo gestantes e lactantes. Foram coletados dados demográficos (data de nascimento, sexo), informações sobre o consumo de água por dia ( $\mathrm{mL} /$ litros/dia), de refrigerantes por semana (garrafas/latas por semana). Considerou-se como ingestão adequada (Al) de água, a recomendação de $2,7 \mathrm{~L}$ para mulheres e $3,7 \mathrm{~L}$ para homens (NAP, 2005) para indivíduos entre 19 a 70 anos de idade.

A análise do teor de $\mathrm{Na}^{+}$, das diferentes marcas de água engarrafada sem gás e refrigerante foi determinada pela análise dos rótulos das garrafas. Nos refrigerantes, além do teor de $\mathrm{Na}^{+}$, foi verificada a quantidade de açúcares. Foram considerados os valores em um litro de água e em $200 \mathrm{~mL}$ de refrigerante. Foi calculada a quantidade e o percentual correspondente de sódio e açúcar, com base na recomendação de no máximo $2000 \mathrm{mg} /$ dia de sódio (WHO, 2012) e de $10 \%$ do valor energético total de açúcar, cerca de $50 \mathrm{~g}$ ou $200 \mathrm{kcal}$ para uma dieta de $2.000 \mathrm{kcal} / \mathrm{dia}$ (NAP, 2005). Os dados foram coletados nos meses de outubro, novembro e dezembro de 2017.

Os dados depois de revisados, foram digitados utilizando o software Excel 2010. Realizaram-se duas digitações a fim de que possíveis erros fossem prontamente identificados. Os dados foram analisados por meio de estatística descritiva. O projeto foi aprovado pelo comitê de ética da Unicesumar, sob número do parecer 2.338.464 em 10/2017.

\section{RESULTADOS E DISCUSSÃO}

Dos 65 questionários aplicados, dois foram excluídos, em função da idade dos entrevistados não condizer com aquela pré-estabelecida no estudo. Foram entrevistados, portanto 63 indivíduos, destes 18 (28,6\%) eram homens e $45(71,4 \%)$ mulheres. A média de idade foi de $39,7 \pm 14,3$ anos.

$\mathrm{Na}$ tabela 1 está descrito o comparativo entre a quantidade de $\mathrm{Na}^{+}$presente nas diversas marcas de água engarrafada e refrigerante, por ordem alfabética. As três marcas de água engarrafada que apresentaram os maiores teores de $\mathrm{Na}^{+}$, em ordem decrescente, foram: Badoit $\AA$, com 180,0 mg; Sferrie $\AA$, com 75,8 mg; São Lourenço®, com $36,0 \mathrm{mg}$. O valor médio de sódio por litro correspondeu a 22,7mg. Considerando a recomendação de ingestão de água de 2,7L para mulheres e 3,7L para homens, o consumo desta quantidade/dia das águas avaliadas corresponderia a ingestão aproximada de sódio de $61,3 \mathrm{mg}$ para mulheres $(3,1 \%$ da recomendação diária de sódio/mulheres) e $84,0 \mathrm{mg}$ para homens $(4,2 \%$ da recomendação de sódio para os homens).

Em estudo realizado no Vale do Taquari no Rio Grande do Sul, Mörschbäche et al. (2015) verificaram teores de $\mathrm{Na}^{+}$entre 18,2 e $86,9 \mathrm{mg} / \mathrm{L}$ nas marcas de água 
pesquisadas, com média de $45,2 \mathrm{mg} / \mathrm{L}$, quase o dobro encontrado no presente estudo.

A legislação vigente não estabelece valores máximos de concentração de sódio na água engarrafada, entretanto a resolução no 274/2005 exige que esteja escrito nas garrafas "contem sódio" quando a concentração de sódio for superior a 200mg/L (ANVISA, 2005), contudo nenhuma das marcas analisadas apresentaram a frase no rótulo, por não ultrapassar esse valor.

As três marcas de refrigerante que apresentaram maiores teores de sódio, por ordem decrescente, foram: Fanta Laranja®, Schweppes Citrus $\AA^{\circledR}$, e Sprite Ice Mint®. As marcas avaliadas de refrigerante apresentaram valor médio de sódio de 23,8mg em $200 \mathrm{~mL}$, equivalente a $1,2 \%$ das recomendações de ingestão de sódio para homens e mulheres.

As marcas de refrigerante que apresentaram teores de açúcares superior a $40 \%$ das recomendações diárias de ingestão de açúcares, ou seja, acima de $20 \mathrm{~g}$ a cada 200mL, em ordem decrescente, foram: Ouro Verde Limão®, com 24g; Ouro Verde Citrus $\AA$ e Pepsi®, com 22 g cada; e Coca Cola $\AA$, Fanta Guaraná $\AA$ e Sprite $\AA$, com $21 \mathrm{~g}$ cada. Rauber et al. (2018), verificaram que na Inglaterra a prevalência de pessoas que excedem a quantidade máxima recomendada para açúcar e sódio aumentou em $85 \%$ e $55 \%$, respectivamente.

TABELA 1: Comparação entre a quantidade de $\mathrm{Na}^{+}$presente em diversas marcas de água engarrafada e refrigerantes. Maringá - $P R$.

\begin{tabular}{|c|c|c|c|c|}
\hline $\begin{array}{c}\text { Marcas de } \\
\text { Água }\end{array}$ & $\begin{array}{c}\mathrm{Na}^{+} \\
(\mathrm{mg} / \mathrm{L})\end{array}$ & $\begin{array}{c}\text { Refrigerante } \\
(\mathrm{mg} / 200 \mathrm{~mL})\end{array}$ & $\begin{array}{c}\mathrm{Na}^{+} \\
(\mathrm{mg} / 200 \mathrm{~mL})\end{array}$ & $\begin{array}{l}\text { Açúcares } \\
\text { (g/200mL) }\end{array}$ \\
\hline Acqua Panna® & 6,4 & Coca Cola $\AA$ & 10,0 & 21 \\
\hline Amaß & 11,5 & Coca Cola Zero® & 29,0 & - \\
\hline Badoit® & 180,0 & Fanta Guaraná® & 15,0 & 21 \\
\hline Bioleve Kids $®$ & 3,4 & Fanta Guaraná Zero® & 33,0 & - \\
\hline Cristal ${ }^{\circledR}$ & 9,2 & Fanta Laranja® & 77,0 & 0,9 \\
\hline Crystal® & 17,7 & Fanta Uva® & 24,0 & 18 \\
\hline Fitness® & 1,1 & Guaraná Antártica® & 11,0 & 20 \\
\hline Font Life® & 6,6 & Guaraná Zero- Antártica® & 11,0 & - \\
\hline Nestle® & 4,2 & Itubaina Original® & 12,0 & 20 \\
\hline Ouro Fino® & 1,1 & Itubaina Zero® & 31,0 & - \\
\hline Platina $\mathbb{B}$ & 9,8 & Kuat® & 20,0 & 13 \\
\hline Safiraß & 7,5 & Kuat Zero® & 35,0 & - \\
\hline Santa Rita® & 2,7 & Ouro Verde Citrus ${ }^{\circledR}$ & 21,0 & 22 \\
\hline São Lourenço® & 36,0 & Ouro Verde Limão® & 12,0 & 24 \\
\hline Schin $\mathbb{B}$ & 6,5 & Ouro Verde Tangerina $\triangle$ & 22,0 & 19 \\
\hline Sferrie® & 75,8 & Pepsi® & 5,8 & 22 \\
\hline \multirow[t]{5}{*}{ Voss $\AA$} & 6,8 & Schweppes Citrus $\AA$ & 50,0 & 1,1 \\
\hline & & Soda - Antártica® & 12,0 & 17 \\
\hline & & Sprite $\mathbb{B}$ & 15,0 & 21 \\
\hline & & Sprite Ice Mint® & 38,0 & - \\
\hline & & Sukita Antártica ${ }^{\circledR}$ & 15,0 & 19 \\
\hline $\bar{x}$ & 22,7 & & 23,8 & 17,3 \\
\hline
\end{tabular}

Valores com mais de uma casa após a vírgula foram arredondados de acordo com norma de arredondamento para números decimais.

Fonte: autores (2018). 
$\mathrm{Na}$ tabela 2 está descrito: ingestão de água por homens e mulheres, de acordo com a faixa etária. Pôde-se verificar que a ingestão de água pelos homens é superior a ingestão das mulheres, entretanto as necessidades de água estabelecidas pela DRIs para homens nas faixas etárias da pesquisa é de um litro a mais que aquelas para as mulheres (NAP, 2005), sendo assim a porcentagem de adequação da ingestão na faixa etária de 19-50 anos foi similar para ambos os sexos. Os homens entrevistados, com idade entre 51-70 anos, ingerem bem menos água que as mulheres, da mesma faixa etária. Nesse sentido, ambos os sexos, necessitam aumentar a ingestão para atingir as recomendações e não ter prejuízos para a saúde.

TABELA 2: Distribuição dos indivíduos, segundo sexo, grupo etário, média de ingestão $(\bar{x})$ per capta de água por dia e \% de adequação em relação a recomendação.

\begin{tabular}{l|cccc}
\hline $\begin{array}{l}\text { Sexo e grupo etário } \\
\text { Mulheres }\end{array}$ & $\mathrm{N}(63)$ & $\overline{\mathrm{x}}(\mathrm{mL})$ & $\begin{array}{c}\text { Recomendação } \\
\text { DRIs }(\mathrm{mL})\end{array}$ & \% adequação \\
$\begin{array}{l}19-30 \mathrm{a} \\
31-50 \mathrm{a}\end{array}$ & 15 & 1.300 & $2.700^{*}$ & 48,2 \\
$51-70 \mathrm{a}$ & 20 & 1.200 & $2.700^{*}$ & 44,4 \\
$\quad$ Homens & 10 & 1.400 & $2.700^{*}$ & 51,9 \\
$19-30 \mathrm{a}$ & 3 & 1.800 & $3.700^{*}$ & 48,7 \\
$31-50 \mathrm{a}$ & 12 & 1.700 & $3.700^{*}$ & 46,0 \\
$51-70 \mathrm{a}$ & 3 & 600 & $3.700^{*}$ & 16,2 \\
\hline
\end{tabular}

DRIs - Dietary Recomended Intake

${ }^{*}$ Adequate Intake (Al) - ou ingestão adequada.

$\mathrm{N}$ : número de entrevistados na pesquisa.

Fonte: autores (2018).

No gráfico 1 esta apresentada a distribuição percentual de ingestão de refrigerante por semana, segundo sexo. A ingestão total na semana pelos entrevistados foi de 44,03L, com um total per capta na semana de aproximadamente $700 \mathrm{~mL}$, sendo ingerido $100 \mathrm{~mL}$ por dia, pouco mais de meio copo americano.

Considerando o refrigerante com maior teor de açúcar $(24 \mathrm{~g} \mathrm{em} 200 \mathrm{~mL})$, a ingestão diária de $100 \mathrm{~mL}$, corresponderia, em média, a $12 \mathrm{~g}$ de açúcar por dia, o equivalente a $24 \%$ da recomendação total de açúcar diário. E ainda, considerandose que a grande maioria dos entrevistados consomem refrigerante apenas nos finais de semana, a ingestão máxima de refrigerante, para não ultrapassar a recomendação de ingestão de açúcar, não deve ser superior a $500 \mathrm{~mL}$ ou aproximadamente 2,5 copos americanos.

Entre 2007 e 2017, o consumo de refrigerante entre a população brasileira caiu 52,8\% (BRASIL, 2018c). No estudo realizado por Claro et al. (2015) com base na Pesquisa Nacional de Saúde realizada em 2013, 23,4\% da população consumia refrigerante ou suco artificial e $53,5 \%$ ingeriam refrigerante pelo menos duas vezes por mês. Já a pesquisa realizada em Nova Boa Vista (RS), em 2013, verificou que $76,3 \%$ da população hipertensa e diabética consome refrigerantes (DESTRI et al., 2017), fato este preocupante, tendo em vista que este é um grupo que deveria reduzir o consumo. 


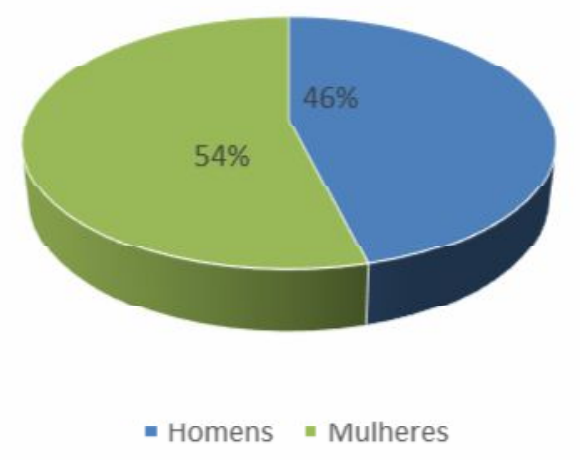

GRÁFICO 1: Consumo de refrigerante por semana, segundo o sexo. Fonte: autores (2018).

\section{AGRADECIMENTOS}

Ao Centro Universitário de Maringá-UNICESUMAR pela concessão da bolsa PIBIC e ao Instituto Cesumar de Ciência, Tecnologia e Inovação - ICETI.

\section{CONCLUSÃO}

As marcas de água e de refrigerante apresentaram variações importantes nos teores de $\mathrm{Na}^{+}$e $\mathrm{CHO}$, mostrando a importância da leitura e verificação dos rótulos das diferentes marcas de água e de refrigerante, tanto em relação ao teor de sódio quanto ao de açúcar, tendo em vista os malefícios à saúde que o consumo excessivo pode causar.

A ingestão de açúcar por meio do consumo de refrigerantes apresentou resultados preocupantes, uma vez que o consumo de 2,5 copos (aproximadamente $500 \mathrm{~mL}$ ) dos refrigerantes que possuem quantidades de açúcares superiores a $20 \mathrm{~g} / 200 \mathrm{~mL}$ atingirão a recomendação máxima de consumo de $\mathrm{CHO}$ por dia. Este fato é especialmente relevante tendo em vista o aumento do excesso de peso e obesidade observado no país.

\section{REFERÊNCIAS}

AFSHIN, A.; MICHA, R.; WEBB, M.; CAPEWELL, S.; WHITSEL, L. et al. Effectiveness of dietary policies to reduce noncommunicable diseases. In: PRABHAKARAN, D. et al. (coord.) Cardiovascular, respiratory, and related disorders. 3. ed. Washington -DC: The World Bank, 2017, v. 5. Disponível em: https://www.ncbi.nlm.nih.gov/pubmed/30212072

ANVISA. Agência Nacional de Vigilância Sanitária. Resolução - RDC no 54, de 15 de junho de 2000. Dispõe sobre o Regulamento Técnico para Fixação de Identidade e Qualidade de Água Mineral Natural e Água Natural. Diário Oficial da União, Poder Executivo, Brasília, DF, 19 jun. 2000.

ANVISA. Resolução no 274, de 22 de setembro de 2005. Aprova o "Regulamento técnico para águas envasadas e Gelo". Diário Oficial da União, Poder Executivo, Brasília, DF, 23 set. 2005.

BRASIL. Departamento Nacional de Produção Mineral. Sumário Mineral 2015. ENCICLOPÉDIA BIOSFERA, Centro Científico Conhecer - Goiânia, v. 15 n.28; p.1159 
Ministério de Minas e Energia, Brasília, v.35, mar. 2016a. Disponível em: http://www.dnpm.gov.br/dnpm/publicacoes/serie-estatisticas-e-economiamineral/sumario-mineral/sumario-mineral-brasileiro-2015

BRASIL. Ministério da Saúde. Com obesidade em alta, pesquisa mostra brasileiros iniciando vida mais saudável. Ministério da Saúde, Brasília, 2018c. Disponível em http://portalfns.saude.gov.br/slideshow/2130-com-obesidade-em-altapesquisa-mostra-brasileiros-iniciando-vida-mais-saudavel

BRASIL. Ministério da Saúde. Promoção da saúde: redução de sódio nos alimentos processados. Ministério da Saúde, Brasília, DF, jun. 2016b. Disponível em: http://portalarquivos2.saude.gov.br/images/pdf/2016/junho/29/ApresentacaoColetiva-do-Sodio-jun2016.pdf

BRASIL. Ministério Da Saúde. Secretaria de Vigilância em Saúde. Departamento de Vigilância de Doenças e Agravos não Transmissíveis e Promoção da Saúde. Relatório do III fórum de monitoramento do plano de ações estratégicas para o enfrentamento das doenças crônicas não transmissíveis no brasil, Brasília - 13 e 14 de agosto de 2013. Ministério da Saúde, Brasília, 2018a. Disponível em: http://portalarquivos2.saude.gov.br/images/pdf/2018/junho/19/RELATORIO-III-

FORUM-DCNT-v-eletronica-13jun18-isbn.pdf

BRASIL. Ministério da Saúde. Vigitel Brasil 2017: vigilância de fatores de risco e proteção para doenças crônicas por inquérito telefônico: estimativas sobre frequência e distribuição sociodemográfica de fatores de risco e proteção para doenças crônicas nas capitais dos $\mathbf{2 6}$ estados brasileiros e no Distrito Federal em 2017. Secretaria de Vigilância em Saúde, Brasília, DF, 2018b. Disponível em: http://bvsms.saude.gov.br/bvs/publicacoes/vigitel_brasil_2017_vigilancia_fatores_ris cos.pdf

CLARO, R. M.; SANTOS, M. A. S.; OLIVEIRA, T. P.; PEREIRA, C. A.; SZWARCWALD, C. L. et al. Consumo de alimentos não saudáveis relacionados a doenças crônicas não transmissíveis no Brasil: pesquisa nacional de saúde, 2013. Epidemiologia e Serviços de Saúde, v. 24, n. 2, p. 257-265, 2015. Disponível em: <http://www.scielo.br/pdf/ress/v24n2/2237-9622-ress-24-0200257.pdf>. Doi: $10.5123 / S 1679-49742015000200008$

COUTO, S.F.; MADRUGA, S.W.; NEUTZLING, M.B.; SILVA, M.C. Frequência de adesão aos "10 passos para uma alimentação saudável" em escolares adolescentes. Revista Ciência \& Saúde Coletiva, v. 19, n. 5, 2014. Disponível em: http://www.scielo.br/pdf/csc/v19n5/1413-8123-csc-19-05-01589.pdf

CUPPARI, Lilian. Guia de nutrição: clínica no adulto. 3. ed., Barueri-SP: Manole, 2014.

DESTRI, K.; ZANINI, R. V.; ASSUNÇÃO, M. C. F. Prevalência de consumo alimentar entre hipertensos e diabéticos na cidade de Nova Boa Vista, Rio Grande do Sul, Brasil, 2013. Epidemiologia e Serviços de Saúde, v. 26, n. 4, p. 857-868, 2017. Disponível em: https://www.scielosp.org/article/ress/2017.v26n4/857-868/ 
FRANCO, G.. Tabela de composição química dos alimentos. 9. ed. Rio de Janeiro: Atheneu, 2001.

MALTA, D. C.; FRANÇA, E.; ABREU, D. M. X.; PERILLO, R. D.; SALMEN, M. C. et al. Mortality due to noncommunicable diseases in Brazil, 1990 to 2015, according to estimates from the global burden of disease study. São Paulo Medical Journal, v. 135, n. $3, \quad$ p. 213-221, 2017. Disponível em $<$ http://www.scielo.br/scielo.php?script=sci_arttext\&pid=S1516$31802017000300213 \&$ Ing=en\&tIng=en>. Doi: $10.1590 / 1516-3180.2016 .0330050117$

MALTA, D. C.; SILVA JR, J. B. O plano de ações estratégicas para o enfrentamento das doenças crônicas não transmissíveis no Brasil e a definição das metas globais para o enfrentamento dessas doenças até 2025: uma revisão. Epidemiologia e Serviços de Saúde, v. 22, n. 1, p. 151-164, 2013. Disponível em: <http://www.scielo.iec.gov.br/scielo.php?script=sci_arttext\&pid=S1679-

49742013000100016>. Doi 10.5123/S1679-49742013000100016

MÖRSCHBÄCHER, A. P.; SILVA, A. M.; SOUZA, C. F. V. Determinação do teor de sódio, potássio e cálcio em amostras de água mineral comercializadas no Vale do Taquari, RS. Revista Destaques Acadêmicos, v. 7, n. 4, 2015. Disponível em: http://www.univates.br/revistas/index.php/destaques/article/view/507/499

NAP. The National Academy Press. Dietary reference intakes for energy, carbohydrate, fiber, fat, fatty acids, cholesterol, protein, and amino acids. Panel on Macronutrients, Panel on the Definition of Dietary Fiber, Subcommittee on Upper Reference Levels of Nutrients, Subcommittee on Interpretation and Uses of Dietary Reference Intakes, and the Standing Committee on the Scientific Evaluation of Dietary Reference Intakes. Institute of Medicine of the national academies, Washington, $\quad$ D.C, $2005 . \quad$ Disponível em: http://www.nationalacademies.org/hmd/ /media/Files/Activity\%20Files/Nutrition/DRITables/5Summary\%20TableTables\%2014.pdf?la=en

RAUBER, F.; LOUZADA, M. L. C.; STEELE E. M.; MILLETT, C.; MONTEIRO, C. A. et al. Ultra-Processed Food Consumption and Chronic Non-Communicable Diseases-Related Dietary Nutrient Profile in the UK (2008-2014). Nutrients, v. 10, n. 5, p. 587, 2018. Disponível em: <http://www.mdpi.com/2072-6643/10/5/587/htm>. DOI: $10.3390 /$ nu10050587

RODWAN JR, J. G. BOTTLED WATER - 2017 - Staying Strong. International Bottled Water Association, v. 1, p. 18, 2018. Disponível em: https://www.bottledwater.org/public/BMC2017_BWR_StatsArticle.pdf

SILVA, S. M. C. S.; MURA, J. D. Tratado de alimentação, nutrição \& dietoterapia. São Paulo: Payá, 2016.

TORTORA, G. J.; DERRICKSON, B. Corpo Humano: Fundamentos de Anatomia e Fisiologia. Artmed Editora, 2016.

WHO. Global Health Estimates 2016: Deaths by Cause, Age, Sex, by Country and by Region, 2000-2016. World Health Organization, Geneva, 2018a. Disponível em 
http://www.who.int/gho/ncd/mortality_morbidity/ncd_total/en/

WHO. Noncommunicable diseases country profiles 2018. World Health Organization, Geneva, v1, p. 51, 2018b. Disponível em http://apps.who.int/iris/bitstream/handle/10665/274512/9789241514620eng.pdf?ua $=1$

WHO. Obesity and overweight. World Health Organization, Geneva, fev. 2018c. Disponível em: http://www.who.int/news-room/fact-sheets/detail/obesity-andoverweight

WHO. Second report of Committee A. World Health Organization, Geneva, n. $65 / 54$, p. 1-3, 25 mai. 2012. Disponível em: http://apps.who.int/gb/ebwha/pdf_files/WHA65/A65_54-en.pdf 